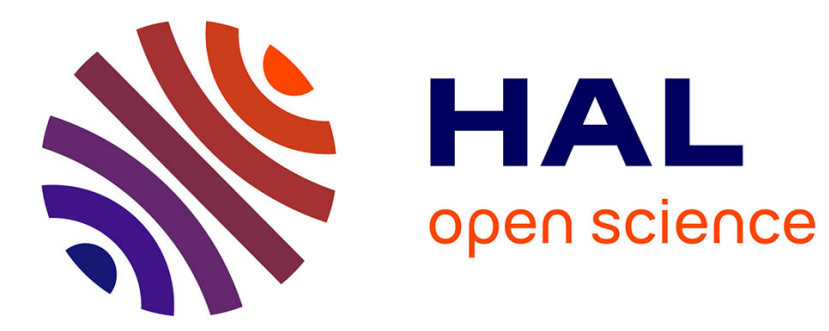

\title{
Time and space-resolved 3D temperature/out-of-plane displacement measurements for investigating the fire behaviour of composite materials
}

\author{
Gillian Leplat, Yves Le Sant, Philippe Reulet, Thomas Batmalle
}

\section{To cite this version:}

Gillian Leplat, Yves Le Sant, Philippe Reulet, Thomas Batmalle. Time and space-resolved 3D temperature/out-of-plane displacement measurements for investigating the fire behaviour of composite materials. Fire and Materials, 2021, 45 (3), pp.435-447. 10.1002/fam.2937 . hal-03176102

\author{
HAL Id: hal-03176102 \\ https://hal.science/hal-03176102
}

Submitted on 22 Mar 2021

HAL is a multi-disciplinary open access archive for the deposit and dissemination of scientific research documents, whether they are published or not. The documents may come from teaching and research institutions in France or abroad, or from public or private research centers.
L'archive ouverte pluridisciplinaire HAL, est destinée au dépôt et à la diffusion de documents scientifiques de niveau recherche, publiés ou non, émanant des établissements d'enseignement et de recherche français ou étrangers, des laboratoires publics ou privés. 


\title{
TIME AND SPACE-RESOLVED 3D TEMPERATURE / OUT-OF-PLANE DISPLACEMENT MEASUREMENTS FOR INVESTIGATING THE FIRE BEHAVIOUR OF COMPOSITE MATERIALS
}

\author{
Gillian LEPLAT $^{1}$, Yves LE SANT ${ }^{2}$, Philippe REULET ${ }^{1} \&$ Thomas BATMALLE $^{1}$ \\ ${ }^{1}$ ONERA / DMPE, Université de Toulouse, F-31055 Toulouse, France \\ ${ }^{2}$ DAAA, ONERA Université de Paris Saclay, F-92190 Meudon, France
}

\begin{abstract}
Main Message. Fire-induced decomposition of composite materials involves complex and coupled multi-physics phenomena experienced for instance in standard tests (cone calorimeter, FAR 25.856(b):2003 and ISO 2685:1998(e)). It is proposed to address this issue in a simplified laboratory facility using a gas burner embedded with coupled measurement techniques to assess all desired physical quantities. A focus is given in this study on non-intrusive (no coating required) digital image correlation (DIC) and infra-red thermography (IRT) measurements. Out-of-plane displacement and temperature of the unexposed surface were accurately measured as a function of time to investigate the 3D fire behaviour of a composite laminate used in the aircraft industry. Those complementary measurements are carried out on a purpose-made experimental set-up in order to provide relevant and correlated information from FOLKI-D algorithm and to identify the damage mechanisms.
\end{abstract}

\section{INTRODUCTION}

\section{Context and fundamental issues}

A significant part is now given to CFRP-based composite materials in the aircraft industry. Composite materials are currently replacing some metallic alloys to provide efficient and resilient structural components such as fuselage and engine carters.. Safety regulations require that structural response and integrity must be addressed to assess the fire performance of materials used where a fire hazard exists. Although standard tests such as FAR 25.856(b):2003 and ISO 2685:1998(e) are and will remain mandatory to evaluate whether the material can withstand the fire threat or not, computational fire models have been significantly improved and can now help understanding the mechanisms by which the fire event is developing and the material is consequently decomposing.

However promising such numerical tools may be, models require an appropriate validation process in order to avoid hazardous and unsafe extrapolations. All too often, numerical results are just compared to experimental measurements on complex configurations to evaluate if the general behaviour or trend is well captured by simulation. Since the material behaviour is driven by multi-physics phenomena (combustion, turbulence, heat transfer, pyrolysis, low Reynolds number flows within porous media, mechanical damage, ...), models validation must be both addressed through the validation of each separate model involving a specific physics and through all interactions and couplings with the other physics.

Standard fire tests involving aeronautical structures take a kerosene burner as a pool fire surrogate to simulate a fire resulting from fuel leakage on the ground after a crash. The fire exposing the lower half of the fuselage structure is replicated in a laboratory by tilting the burner and the test coupon. This test is defined in FAR 25.856(b):2003 standard regulation to evaluate whether the material can withstand the fire and avoid burn-through to prevent the flame penetration into the cabin. Two criteria must be fulfilled for the test to pass: no burn-through and the heat flux threshold not crossed on the backside. Composite materials generally do not experience burn-through after 5 minutes or more of fire exposure, even for thin coupons. This property is brought by the reinforcement of carbon fibres that remain inert even in such harsh environment. However, the matrix is subjected to chemical 
transformations occurring as the temperature increases within the material. The resin of CFRP (Carbon Fibre Reinforced Polymer) or OMC (Organic Matrix Composite) materials experiences pyrolysis and decomposes into a solid residue named char associated with gas release. The gas phase is produced from the reaction area, transported within the porous material and released out of the material surface. This off-gassing phenomenon is occurring first on the side exposed to fire because pyrolysis makes porosity increase and permeability increases as a consequence. Fire tests performed on composite materials usually exhibit ignition of the gas phase (see photographs on figure 1). An energetic contribution is then added by the material to the heat flux induced by the burner flame. For longer fire exposures or thinner materials, off-gassing can also occur on the unexposed surface even though ignition is not likely to occur without an ignition source because temperature remains low enough to prevent self-ignition.

\section{Motivation and technical positioning}

Qualitatively, the observation of fire standard tests shows a complex fire development on the exposed surface of the test coupon that becomes even more complex when the gas phase is released. Quantitatively, it is almost impossible to dissociate the contribution of each phenomenon into the global material reaction to fire. The optimisation of materials and structures with respect of the fire criterion is limited because of the lack of information about the driving processes.

Aircraft and passengers safety is granted by standard tests. Trying to understand and optimize the fire performance of materials and structures only from standard tests would be too difficult and expensive for manufacturers. Scientific approach usually recommends taking a step back to broaden the perspective and to address the problem from the basis. The material thermochemical behaviour can be assessed through TGA ${ }^{1}$ and DSC analysis at the condensed matter scale. Pyrolysis model is then developed from identified kinetic parameters associated with the enthalpy for each macroscopic reaction occurring successively.

However, the behaviour at the matter scale cannot be extrapolated to the behaviour observed during standard tests. A step further in representativeness shall consider the material scale taking into account heat transfer and thermo-mechanical behaviour. The approach developed at ONERA chose to sweep aside the fire at this scale to consider the behaviour of the material itself without any contribution of the flame. Indeed, the material behaviour is the response to the heat flux on its surface balanced with thermal loss with the ambient environment. Not only is the heat flux distribution very difficult to assess but the heat flux evolution following the gas phase ignition significantly increases the uncertainties in the results analysis. Moreover, exposing a composite test coupon to fire at the material scale (usually up to $10 \mathrm{~cm}$ characteristic length) results in applying a uniform heat flux onto the material surface. It is important to keep in mind that composite materials and especially laminates have orthotropic properties with in-plane thermal conductivity 5 or 10 times higher than in the through-thickness direction. A uniform heat flux will only reveal the behaviour in that direction. ONERA has thus developed a test facility named BLADE (which can be translated as Laser-induced characterisation and decomposition) to assess thermal properties of anisotropic materials and non-intrusively analyse the material behaviour resulting from the decomposition induced by a pure radiative laser source in a confined and controlled environment ${ }^{2,3}$. The thermal response measured on the unexposed surface in such conditions is driven by heat transfer within the material and decomposition processes affecting the material properties and the energy balance. The heat exchanges between the test coupon surfaces and the surrounding environment is reduced to radiation heat transfer since convection is avoided by lowering the internal pressure of the test chamber to $5 \mathrm{mBar}$. It provides relevant conditions to understand the material behaviour and to build database for model validation. But this validation step is limited by the mechanical damage occurring within composite coupon even without any fire. Delamination is observed in the experiments and the effect on the thermal response is quantitatively evaluated. However, it is impossible to implement measurement techniques to characterise the delamination during the experiment in such confined environment.

Although fundamental results concerning the thermal behaviour and the damage mechanisms are built upon laser tests, complementary analyses are required to take into account all contributions at stake for evaluating the fire performance of an aircraft material. The next steps in representativeness are firstly 
to increase the scale to take into account structural behaviour and to investigate delamination onset and growth within composite laminates. Secondly, fire tests shall be performed in the laboratory ambient environment as in standard tests to include convective loss. Thirdly, fire tests cannot be performed without fire. But fuel burners generate a complex flame resulting from the evaporation of kerosene liquid droplets by the igniter and the combustion with the swirled air flow in the burner cone. Combustion of kerosene in such conditions creates soot particles that significantly increase the radiative contribution on the global heat flux and turn the flame to yellow. The approach of fire testing at ONERA is based on a gas burner that generates a premixed flame in order to accurately control the air/fuel ratio. The heat flux is voluntarily focused on the centre of the exposed surface of the test coupon with a nonuniform distribution for the anisotropic behaviour of the material to be measured. Composite laminates have an intrinsic 3D thermal and mechanical behaviour. As a consequence, 3D behavioural models must be developed to accurately predict the material reaction to fire in standard test although the test configuration provides uniform surface conditions and an expected 1D behaviour in the throughthickness direction. However, observations show that major phenomena are occurring on the edge of the test coupon, especially for composite materials. Indeed, the use of a coupon frame holder, usually associated with an insulating material in between, affects the material behaviour during the experiment. Heat transfer is significantly reduced in this area and the coupon is mechanically constrained by the frame. Moreover, in cone calorimeter experiments, similar edge effects are observed with off-gassing on the edges and ignition of volatiles in this area while the heat flux is considered to be only applied on the exposed surface. The interpretation of test results is distorted and the one-dimensional behaviour hypothesis is no longer valid.

\section{Background and proposed approach}

Thermomechanical investigations of composites usually require measuring both the temperature and the displacement of the test coupon exposed to fire. The two often used measurement techniques are infrared thermography ${ }^{2,3}$ and digital image correlation ${ }^{4}$ in order to assess full field data compared to measurements with physical sensors or some other optical techniques where only area-average data are assessed. The challenge then mainly resides in combining the two techniques to obtain simultaneous time-resolved distributions of temperature and displacements in the region of interest. Cholewa et al. ${ }^{5}$ proposed in 2016 a novel technique to couple DIC performed with commercial software with IRT measurements. The technique was named TDIC. The IRT and DIC cameras were calibrated with the standard pin-hole stereo model accounted for temperature-dependency of the emissivity of the paints used for the speckle pattern coating on the test coupon surface. Such method was immediately and successfully applied by Hodges et al. for the thermomechanical analysis of a structural I-beam exposed to fire ${ }^{6}$ and for resolving obstructed views on a stiffened panel using multiple TDIC systems ${ }^{7}$ in order to combine the different fields of view into a single dataset to validate finite-elements (FE) simulations.

Nevertheless, the TDIC technique, as well as conventional DIC technique, relies on a coating applied onto the material surface where measurements are performed. High contrast is required for the speckle pattern painted on the test coupon while surface emissivity must be determined for IRT. Cholewa et al. ${ }^{5}$ developed a dedicated procedure to characterise the paint optical properties based on the grey body assumption and diffuse surface radiation. However authors show that negligible effect of the speckle pattern on the IRT measurements can be obtained, temperature measurement accuracy is generally granted if the surface optical properties of the material surface are known as a function of all driving parameters for the conversion in temperature from the luminance measured by the IRT camera. The assessment of the hemispherical emissivity is usually recommended if the camera if oriented in the perpendicular direction of the test coupon surface. If the camera orientation is not normal to the material surface, emissivity must be determined as a function of the angle. But in both situations, emissivity is a spectral property and values as a function of the wavelength and integrated with respect to the camera spectral range. The spectrum must exhibit negligible change along the spectral range for measurements to be reliable as a function of temperature. As a consequence, using different paints to apply the speckle pattern may induce bias on IRT measurements without being noticed if constant values are assumed. For these reasons, a special attention is to be given on coating characterisation to avoid limitation in the result analysis. 
The proposed approach is based on non-intrusive IRT and DIC measurements for both the temperature and the displacement. Indeed, if coating is required for reflective metallic material for instance, a black paint is used and the spectral emissivity is assessed and integrated for quantitative IRT measurements. For composite materials, the optical properties usually exhibit high values of emissivity and absorptivity on the material virgin surface. A few percent increase is even noticed when the material is charring due to thermal decomposition. When exposed to fire, the absorptivity and emissivity of the front surface will be affected but the unexposed surface will not for moderate exposure time and thickness. If surface emissivity varies as a function of temperature and decomposition growth rate are significant, temperature conversion from the luminance signal measured by the IRT camera become complicated and it must be iteratively resolved since temperature and local emissivity are initially unknown. For test cases presented hereafter, either the material does not decompose (metallic materials) or the unexposed surface where measurements are performed remains uncharred (composite materials).

On the basis of these observations and limitations, ONERA proposed another test facility embedded with extensive measurement techniques to help understanding composite material reaction to fire and to bridge the gap towards standard tests and certification.

\section{EXPERIMENTAL APPROACH}

\section{FIRE facility}

The FIRE facility was mainly developed for composite applications. The material reaction to fire requires investigating both exposed and unexposed surfaces with combined measurements techniques in order to include all physical phenomena occurring during the experiment. FIRE stands for Flamewall Interaction Research Experiment. The facility focuses on the effect of the gas phase released by the material decomposition in the combustion processes on the side exposed to fire. Concurrently, some composite materials may experience mechanical damage. Composite laminates are usually subjected to delamination that affects heat transfer in the through-thickness direction with noticeable effects on the unexposed surface.

The test facility, illustrated in figure 1, is composed of 3 major parts or components assembled with aluminium profiles:

1. The test coupon is located at the centre of the support frame. It is aligned horizontally and surrounded by a ceramic refractory material made of calcium silicate. The support frame is totally disconnected from the test coupon to eliminate interference and to perform mass loss measurements.

2. A large gas burner generates a premixed air-propane flame out of a $\varnothing 40 \mathrm{~mm}$ outlet diameter. The exposure time is controlled by moving the burner on a linear sliding rail with a pneumatic piston actuator. The burner is initially out of the support frame during thermal stabilisation after flame ignition. Burnt gases are extracted through an exhaust hood located at the ceiling of the laboratory. The burner is then moved below the test coupon to reach the centre of the exposed surface at the beginning of the experiment.

3. The main frame structure of the facility includes secondary gas exhaust and optical accesses for the measurements on the unexposed surface on the upper chassis and optical accesses for visualisation and flame dynamics measurements on the lower chassis.

\section{Measurement techniques}

The main interest of the FIRE facility is to simultaneously perform different and complementary measurements during the test in order to correlate the dynamic behaviour of the flame with the thermal, chemical and mechanical behaviours of the material exposed to fire. The key feature is that all measurements are non-intrusive so that the behaviour of both fire and material remains unaffected.

Two flowmeters control the mass flow rate of air and propane injected in the burner. The gas mixture is homogenised through a bed of glass beads and a stainless steel honeycomb before being transported towards the burner outlet. Temperature measurements with K-type thermocouples ensure that the burner stabilised wall temperature is reached before the experiment to begin. This process takes up to 
60 minutes to converge and it is mandatory for reproducibility of the experiment to be granted.

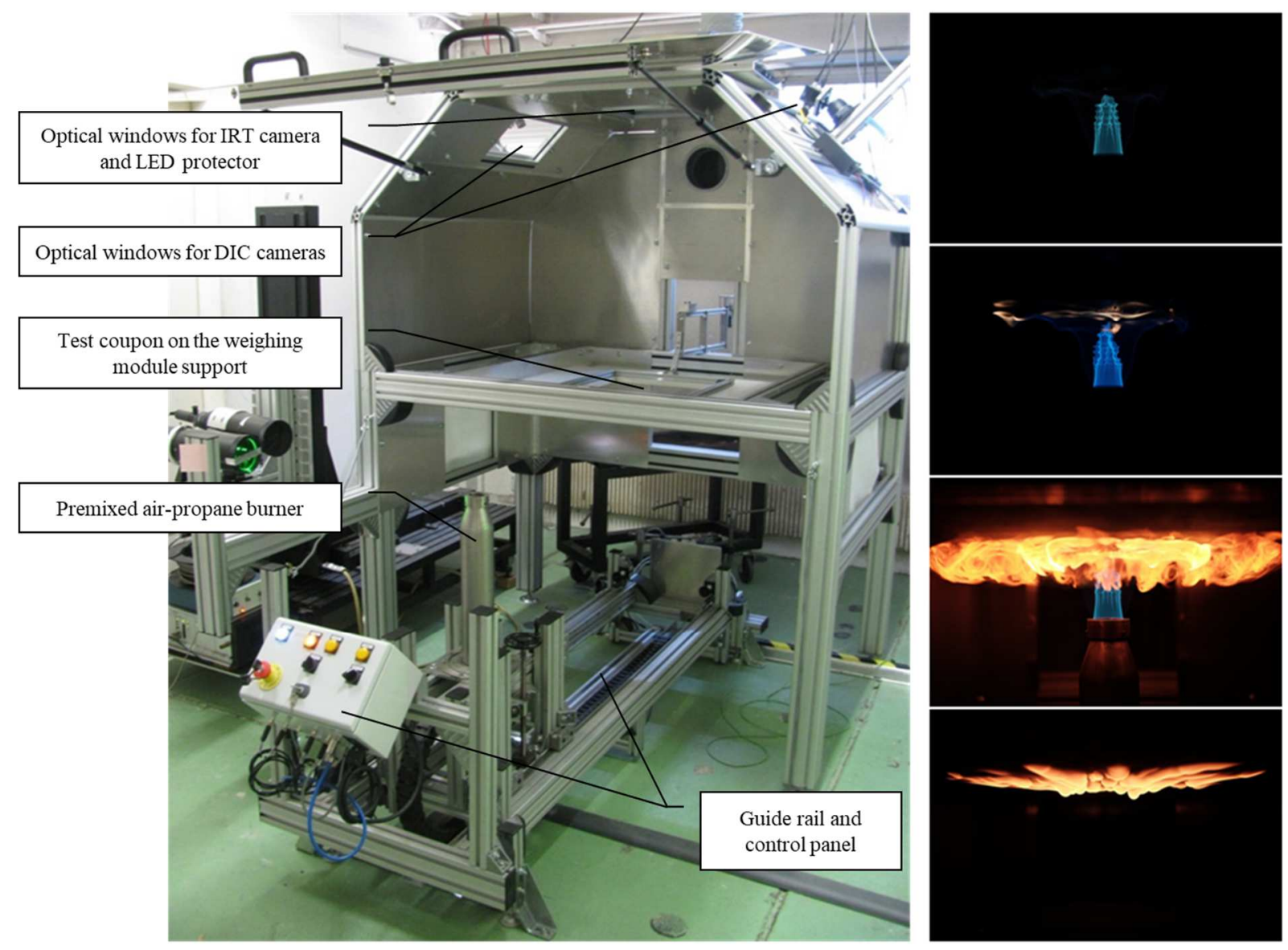

Figure 1 - FIRE facility and photographs of composite laminate reaction to fire

Position sensors on the linear sliding rail of the burner record when the burner leaves the initial station and when it reaches the centre of the test coupon. The signals are used for measurements synchronisation.

The upper chassis of the frame structure of the facility includes:

- A secondary extraction system for gas exhaust: burnt gas from the burner when the test coupon is exposed to fire as well as smokes and fumes released by the material decomposition are extracted. Gas temperature is controlled at the extraction point with a K-type thermocouple.

- $\quad$ Optical access for infra-red thermography measurements through a silver-coated mirror and a ZnSe window in order to isolate the camera from the polluted and hot environment. The optical axis of temperature measurements is aligned perpendicularly to the test coupon surface. The thermography camera is calibrated on the temperature range $\left[20-700^{\circ} \mathrm{C}\right]$ and 4 integration times are used to increase the dynamic range of the camera. The processing of luminance data measured by the camera is carried out with a procedure developed at ONERA. That procedure takes into account the spectral optical properties of the material surface (emissivity and absorptivity), the transmission factor of mirrors and windows through the optical path and the temperature calibration using a black body of both camera and lens used in the experiment. Spectrophotometry is used for the characterization of the reflectance and transmittance of the material surface as a function of the wavelength of the source. Two different sources are combined to cover the spectral range from $250 \mathrm{~nm}$ to $21 \mu \mathrm{m}$. For monochromatic heat source such as laser, the absorptivity is obtained from the value measured at the wavelength of the laser. For broadband heat sources such as fire, the grey body hypothesis is assumed where absorptivity is equal to emissivity. For infrared thermographic measurement, the emissivity value is integrated from the spectral emissivity previously measured over the spectral range of the camera sensor, i.e. for the InSb sensor of the FLIR SC7600 series camera, the emissivity is integrated over the $[3-5 \mu \mathrm{m}]$ spectral range. 
- Optical accesses for the two DIC cameras recording images of the material surface in a stereoscopic configuration. The optical axes are tilted with a $30^{\circ}$ angle with respect to the vertical median plane to perform stereovision. Glass windows with anti-reflective coating separate the cameras from the inner side of the frame structure for similar reasons than for the thermography camera.

- Optical access in the vertical direction close to infra-red thermography access for projecting a random speckle pattern onto the material unexposed surface using a high power blue LED. To remain non-intrusive, conventional DIC procedure cannot be carried out because any coating applied onto the material surface will affect the surface emissivity for thermography measurements and may prevent off-gassing on the unexposed surface of decomposition volatiles resulting from the pyrolysis of the composite material.

The geometrical configuration of the optical measurements is presented in the figure 2 below. The three cameras are located in the same vertical plane in the direction perpendicular to the material surface plane. The LED projector is tilted by a $12^{\circ}$ angle behind the IRT camera DIC cameras are tilted by $30^{\circ}$ angle with respect to the vertical direction in order to maximize the field of view.

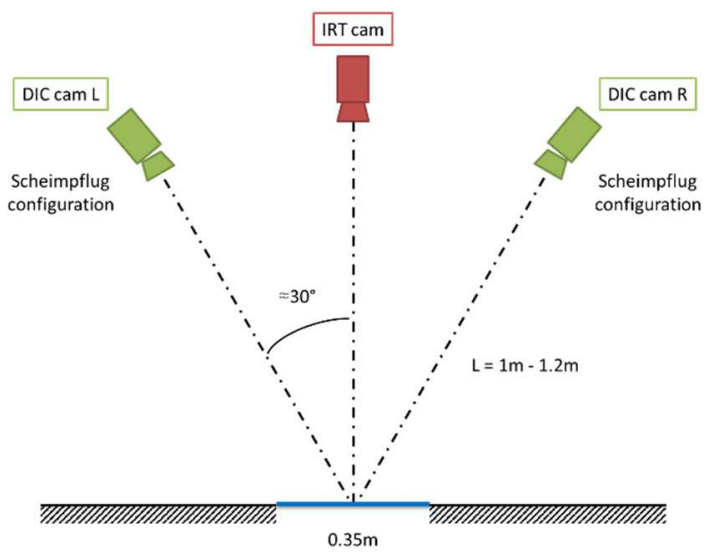

(a) Front view

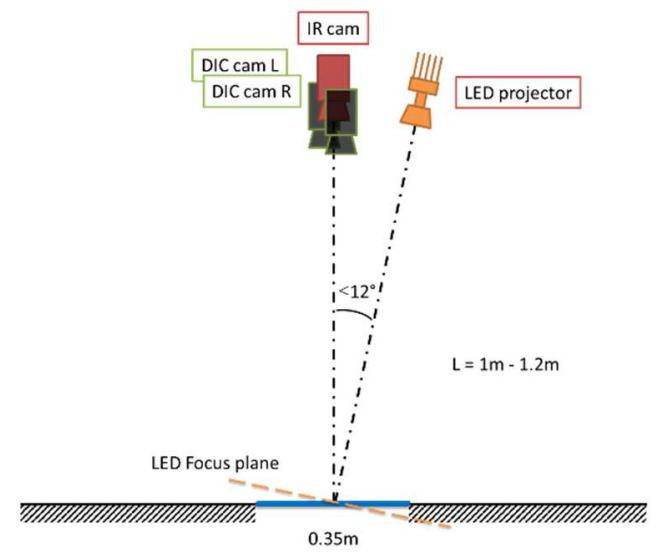

(b) Side view

Figure 2 - Geometrical configuration of the optical measurements in the FIRE facility

The more titled the camera are titled (with an optimal value of $45^{\circ}$ for out-of-plane displacements), the more accurate the displacement will be measured. However, one drawback of this geometrical configuration is that the focus plane of the camera and the material surface plane are not aligned, resulting in blurry pictures. That is why Scheimpflug mounts are installed in DIC cameras in order to tilt the camera sensor and the lens. This technique is always applied when performing stereoscopic PIV (SPIV) measurements to assess flow velocity field in fluid dynamics measurements. In this particular application, the Scheimpflug mount is integrated within the lens using a tilt-shift lens on each camera (such lens is often used by architectural photographers).

The lower chassis of the frame structure includes:

- Three optical accesses for visualisation of phenomena occurring on the exposed surface of the test coupon. A high resolution colour photographic camera is used at the rear of the frame structure while laser measurement techniques can be performed from the sides such as LDV and PIV to characterise flame dynamics.

A high precision weighing module is implemented in the facility but outside the main frame structure to prevent sensor heating. The module is connected to a Roberval-type balance system with minimum contact to limit friction. The other side of the Roberval balance is connected to the coupon holder. Such configuration offers an unobstructed field of view above the test coupon to perform all optical measurements and allows preloading the balance on the module side to compensate for coupon initial mass and holder mass. Another key feature is the use of a high precision electromagnetic compensation weighing module. The measurement principle adjusts the electrical current inside a coil for the magnetic sensor to stay at the same position. As a consequence, the test coupon will not be affected by any undesired displacement during the experiment when mass is lost. Mass loss rate, temperature and displacement of the unexposed surface can thus be performed simultaneously. Moreover, the distance 
between the burner outlet and the material surface will not be affected as well by the mass loss measurements. The exposed surface of the material will remain aligned with the calcium silicate plate in order not to introduce aerodynamic interference on the material edges. A small gap, lower than $1 \mathrm{~mm}$, separates the material outline from the calcium silicate plate to avoid any friction with the balance. This paper is focused on the thermo-mechanical measurement technique development so mass loss rate assessment will not be presented.

The facility operation is fully automated using a Labview-based programme that automatically starts the test and the acquisition of data with all measurements in sync. For safety reasons, unburnt propane detectors are implemented on the ground level and both gas extraction points and connected to the emergency stop function to interrupt the experiment and prevent any hazardous situations by immediately closing the propane gas supply.

\section{Materials}

The investigation deals with a material used in the aeronautical industry for primary and secondary aircraft structures. The T700GC/M21 is a composite laminate consisting of TORAY T700GC carbon fibres and HEXCEL M21 epoxy resin reinforced by thermoplastic nodules. Eight $260 \mu \mathrm{m}$-thick $\mathrm{M} 21 / 35 \% / 268 / \mathrm{T} 700 \mathrm{GC}$ unidirectional $268 \mathrm{~g} / \mathrm{m}^{2}$ prepreg plies are stacked and cured to manufacture the composite laminate. This high fibre mass fraction is the consequence of the stacking of two fibre tows in each prepreg tape. The M21 resin is a tough epoxy matrix and exhibits excellent damage tolerance, especially in the case of high energy impacts. To obtain such properties, it contains thermoplastic nodules around each fibre tow with a characteristic diameter of $25 \mu \mathrm{m}$.

Fibres average diameter is $7 \mu \mathrm{m}$ and the volume fraction of fibres is 0.57 for the cured material. The final material thickness is $2.1 \mathrm{~mm}$. The orientations of the plies in the quasi-isotropic (denoted ISO) layup are:

$$
[45 / 90 /-45 / 0]_{s}
$$

A reference material shall be used for preliminary tests in order to develop the method presented hereafter and to evaluate its capabilities. INCONEL 600 metallic test coupons are thus chosen for their resistance and behaviour in a high temperature environment.

Large test coupons are used for the experiments in the FIRE facility with in-plane dimensions of $350 \times$ $350 \mathrm{~mm}^{2}$.

\section{COMBINING DIC AND INFRA-RED THERMOGRAPHY FOR ASSESSING 3D TEMPERATURE MEASUREMENTS}

The numerical method for processing the experimental data is developed at ONERA by 3 scientific departments of ONERA working together: DTIS (Image Processing and Systems), DAAA (Aerodynamics, AeroAcoustics and Aeroelasticity) and DMPE (Multi-Physics for Energetics). The DIC (Digital Image Correlation) technique presented hereafter, is mainly used by mechanical engineers and scientists. It was derived from a technique used in fluid dynamics applications. The PIV technique, standing for Particle Image Velocimetry, consists in measuring a flow velocity field using images of seeding particles crossing a laser sheet. Such measurements can be performed to assess 3 components of the velocity vector in the region of interest illuminated by the laser sheet. The technique is called Stereo-PIV or SPIV. Actually, DIC and PIV are very similar techniques: the texture induced by seeding particles crossing the laser sheet and recorded by the cameras in PIV applications is simply replaced by a texture projected or painted onto the surface of a material in DIC.

\section{Core principle of FOLKI_SPIV and FOLKI_D: computer vision algorithm FOLKI}

Both FOLKI_SPIV for PIV and FOLKI_D for DIC are based on the algorithm FOLKI. FOLKI is a French acronym that can be translated as Optical Flow with an Iterative scheme based on the LucasKanade (LK) paradigm. The seminal work published by Lucas and Kanade ${ }^{8}$ is widely used by image correlation methods using the block matching principle, i.e. selecting a small interrogation window (IW) in the first image and matching it as best as possible with a window of the same size in the second 
image.

In practice, the LK objective is iteratively solved, within a predictor-corrector scheme. As a direct and naive translation of the global objective into iterations is not stable, some authors proposed to introduce an additional filtering step of the predictor or of the corrector in the iterations. In contrast, the algorithm FOLKI published by Le Besnerais and Champagnat ${ }^{9}$ is naturally stable, and therefore it does not require any filtering.

Consider two images $I_{1}$ and $I_{2}$ to be matched by assessing the displacement field $u$ that minimises the sum of squared differences $\operatorname{SSD}(u)$ :

$$
S S D(u(k))=\sum_{m \in I W}\left[I_{1}(m)-I_{2}(m+u(k))\right]^{2}
$$

IW is the interrogation window centred on pixel $k$. The displacement field at the end of the iteration is denoted $u_{0}$ and $u_{0}$ is then updated in the next iteration by the increment $d u$ using the equation:

$$
\operatorname{SSD}(d u(k))=\sum_{m \in I W}\left[I_{1}(m)-I_{2}^{u_{0}}(m+d u(k))\right]^{2}
$$

where $I_{2}^{u_{0}}=I_{2}\left(m+u_{0}(m)\right)$ is the image $I_{2}$ interpolated using the displacement field $u_{0}$. In the following, only a 1D displacement along $x$ is considered for the sake of simplicity. The writing for 2D or 3D displacements is formally identical. The previous equation can be linearized by introducing the spatial derivative of $I_{2}^{u_{0}}: D_{2 x}=\partial I_{2}^{u_{0}} / \partial x$. Equation [2] now writes:

$$
S S D(d u(k))=\sum_{m \in I W}\left(I_{1}(m)-I_{2}^{u 0}(m)-D_{2 x}(m) d u(k)\right)^{2}
$$

The minimisation of $S S D$ is obtained when the derivative of $S S D$ with respect to $d u(k)$ is equal to zero:

$$
d u(k) \sum_{m \in I W} D_{2 x}(m)^{2}=\sum_{m \in W} D_{2 x}(m)\left(I_{1}(m)-I_{2}^{u 0}(m)\right)
$$

Replacing $d u(k)$ by $u(k)-u_{0}(k)$ provides the updated displacement field $u(k)$ :

$$
u(k) \sum_{m \in I W} D_{2 x}(m)^{2}=\sum_{m \in I W} D_{2 x}(m)\left(I_{1}(m)-I_{2}^{u 0}(m)+D_{2 x}(m) u_{0}(k)\right)
$$

The iterative scheme based on this equation is not stable. To make it stable, $d u(k)$ is replaced by $u(k)-$ $u_{0}(m)$ in FOLKI instead of $u(k)-u_{0}(k)$. The equation is then:

$$
u(k) \sum_{m \in I W} D H_{2 x}(m)^{2}=\sum_{m \in I W} D_{2 x}(m)\left(I_{1}(m)-I_{2}^{u 0}(m)+D_{2 x}(m) u_{0}(m)\right)
$$

The resolution of the iterative process is thus based on the Gauss-Newton (GN) algorithm. A drawback is that the update $d u(k)$ cannot be larger than 2 or 3 pixels, otherwise the linearization in equation [3] is no longer valid. As the displacement in DIC images is usually greater, a multi-resolution scheme is used. An image pyramid is built starting from the recorded raw images, which correspond to the ground level as shown in figure 3. Stepping up a level in the pyramid is done by applying a low-pass filter and a decimation factor 2 . At each level, the displacements are thus divided by a factor 2 , up to the last level where the displacements become lower than 3 pixels and can be truthfully captured by the GN algorithm.

When the displacement field is assessed at the highest level, it is multiplied by two and used as the initial guess for the previous lower level. This process is repeated up to the ground level. 


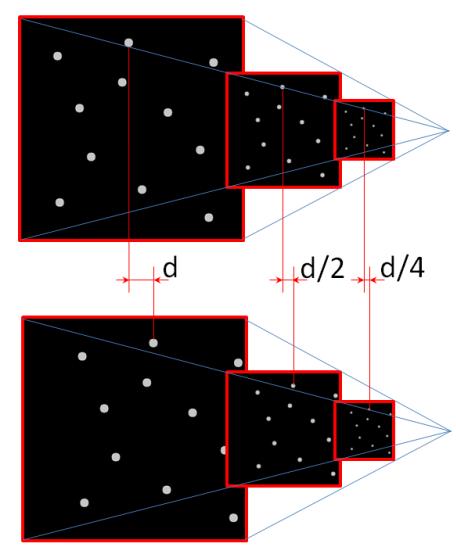

Figure 3 - Image pyramid with two levels, $L=2$

In summary, the algorithm only requires a few input parameters:

1. The size of the $I W$ depending on the image texture. It is usually within the range of [1531 pixels]. Higher sizes are used when the image texture is very low. Lower sizes require a very fine texture.

2. The level number $L$ of the pyramid, understood as the number of levels additionally built from the ground level of the raw image. The level number should be chosen so that the maximum displacement is lower than $2^{\mathrm{L}+1}$. The typical level number for PIV is 3 , enabling to capture displacements lower than $2^{3+1}=16$ pixels. For DIC, a level number of 6 can be used to capture displacements up to $2^{6+1}=128$ pixels. However, it is important to keep in mind that the size of the images at the highest level is the initial size divided by $2^{\mathrm{L}}$. This size is 32 pixels with an initial size of 2048 and a level number of 6 . Using a higher level number does not make sense since the image size at the top level would be lower than the IW size! That is why a dedicated strategy for large displacement is used for DIC.

3. The iteration number. Usually 5 iterations are enough to obtain fully converged results. This number can be increased up to 10 when the convergence is slow, such as when the texture quality is poor.

The key properties of FOLKI compared to conventional PIV or DIC methods are:

1. The update in equation [6] involves the displacements of all the pixels in the IW. As a consequence, the algorithm must compute the displacement at every pixel. It does not mean that the spatial resolution is equal to 1 pixel. The spatial resolution depends on the size of the IW since correlation determines the average displacement of the texture.

2. Equation [6] shows that there are strong interactions between adjacent pixels. This might be an explanation for the regularity of the vector fields observed in practice, making any outlier rejection step useless (see next section for more details).

3. Computations in equation [6] are mainly separable convolutions (sums over the IW).

This last property makes FOLKI very well suited for an implementation on GPU (Graphical Processor Unit) because computations are easily parallelizable. It is then possible to process PIV or DIC images nearly in real time.

\section{A noticeable feature: no filtering}

Consider equation [6] and replace $u(k)$ by $d u(k)+u_{0}(k)$ :

$$
\begin{aligned}
& d u(k) \sum_{m \in I W} D_{2 x}(m)^{2}= \\
& \sum_{m \in I W} D_{2 x}(m)\left(I_{1}(m)-I_{2}^{u 0}(m)\right)+\sum_{m \in I W} \boldsymbol{D}_{2 \boldsymbol{x}}(\boldsymbol{m})^{2}\left(\boldsymbol{u}_{\mathbf{0}}(\boldsymbol{m})-\boldsymbol{u}_{\mathbf{0}}(\boldsymbol{k})\right)
\end{aligned}
$$

This equation is nearly the same as equation [4] (which exhibits a divergent behaviour), with an additional term (in bold), ensuring convergence without the need of any intermediate filtering unlike an important number of algorithms in the scientific literature that rather chose to stick to equation [4]. 


\section{Adaptation for PIV and DIC}

The algorithm FOLKI was designed for image correlation ${ }^{10}$ as used in computer vision. Some adaptations were done to adapt it to PIV and then to DIC images processing:

1. Preliminary zero normalization of the raw images using the IW size. The local average is subtracted and the residual is divided by the local dynamics. Masks are taken into account when computing the convolutions. This enables to compute the displacement field even when the IW contains masked pixels, as on borders.

2. Masks are taken into account at each step of the computational pipeline. Only the useful areas are processed and areas where the texture is too low are automatically rejected. Thus, parts of the images where the IW contains discarded pixels or areas outside of the masks can be processed as well.

\section{Extra features required for DIC}

There are actually three computational steps for DIC instead of only one for PIV:

1. Stereovision on the initial images to compute the initial shape;

2. Stereovision on the final images to compute the final shape;

3. Computation of the displacement field (the only step for PIV).

The same calculation engine is used for these three steps ${ }^{11}$. In the FIRE application, only the first step (computing the coupon shape) is useful since the pattern is projected instead of being coated onto the coupon.

\section{Cameras calibration}

Camera calibration is mandatory for any optical measurements. Calibration must be performed for all cameras in order to assess the required optical parameters: extrinsic parameters (camera location and orientation) and intrinsic parameters (field of view and lens distortion).

The calibration body used for calibrating the camera is a dual plane 3D calibration body used in PIV (figure 4(a)). It consists of a grid array of white dots on a black background distributed on 2 levels allowing 3D calibration from a single view. Fiducial markers are located at the centre of the calibration body to detect the body and its orientation in the field of view. The calibration routine implemented in the software developed at ONERA automatically detects all dots of the calibration body from the selection of the 3 dots surrounding the fiducial markers (markers 0, 1 and 2 in figure 4(a)). The procedure is applied for both DIC cameras.

Cameras calibration in the FIRE facility benefits from the features of such a calibration body because it can be used to calibrate the IR camera as well. The images in the IR spectrum offer an emissivity contrast high enough to detect the dots and fiducial markers. White dots have a lower emissivity than the black background (figure 4(b)). As a consequence, the calibration body image in the IR spectrum shows black dots onto a white background.

However, the image definition from the IR camera $(640 \times 512$ pixels $)$ is lower than the image definition from the DIC cameras $(2456 \times 2058$ pixels $)$ and special care must be taken to select the fiducial markers in the right order (figure 4(b)). Camera linking is enabled by using the same calibration body for DIC and IR cameras: they are calibrated within the same world coordinate system. A single image recorded from the 3 points of view is required to perform the calibration process without moving the calibration body. 


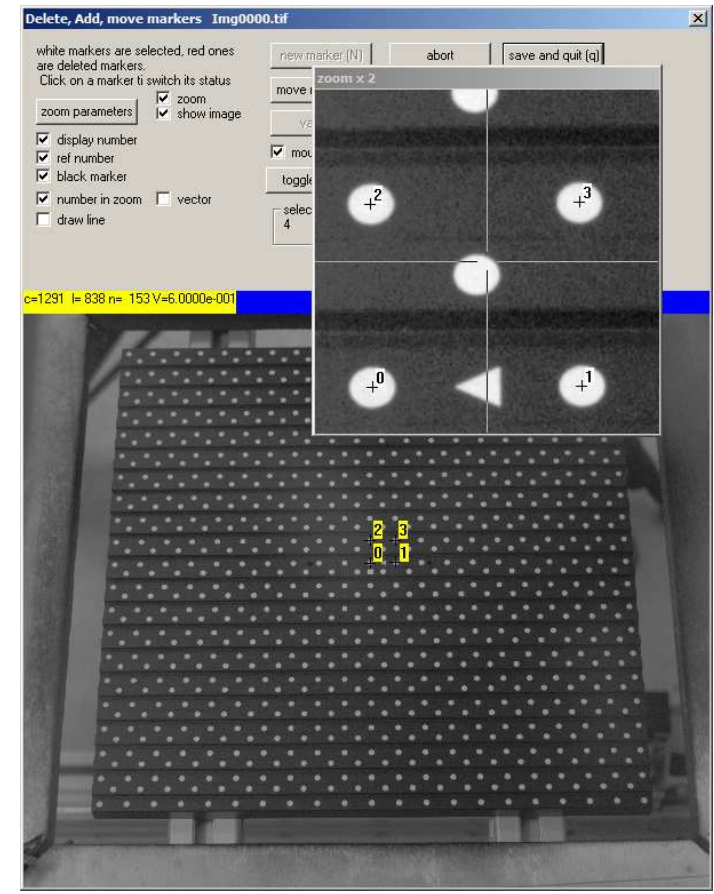

(a) Calibration image from DIC camera

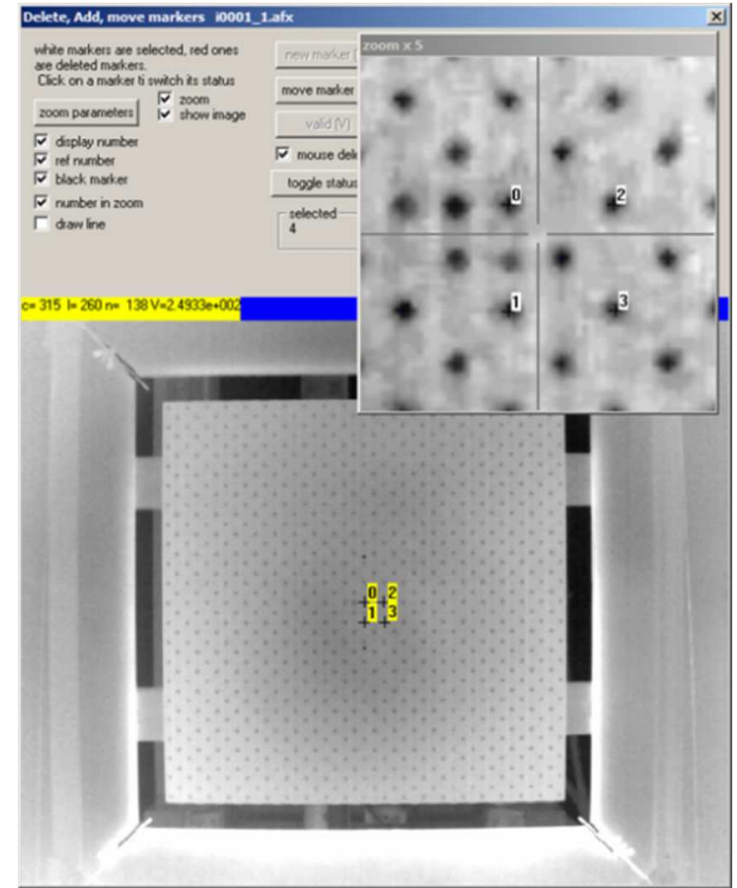

(b) Calibration image from IR camera

Figure 4 - Calibration body and fiducial markers for DIC and IR cameras calibration

\section{Lagrangian tracking of temperature}

The 3D temperature reconstruction from the combination of DIC and IR thermography measurements is also called Lagrangian tracking of temperature. The technique consists in three steps:

1. Applying DIC to provide the shape of the coupon, i.e. the world coordinates of the point computed for every pixel.

2. Projecting the points onto the IR image, using the calibration of the IR camera.

3. Picking the temperature at the projected points and then creating a temperature image of the same size as the DIC images.

Since the image acquisition from all 3 cameras is performed in sync, the results are the coupon shape and the 3D temperature field. This is done at each time step of the experiment. The technique is thus time-resolved.

The Lagrangian tracking of temperature was implemented within FOLKI_D algorithm during this test campaign and this feature is now available in the software.

\section{APPLICATIONS}

The capabilities of the method are presented in the next two sections. Tests were conducted on two different coupons (references are given in the Materials section) to bring out the discrepancies about the reaction to fire of metallic materials and composites laminates used in the aircraft industry. The figures 6 and 7 display:

- upper right side: the instantaneous 3D temperature field on the unexposed surface of the test coupon where the DIC and IR thermography are performed. The vertical coordinate $Z$ is magnified with a ratio $Z / X=Z / Y=2$ for visualisation convenience but the absolute values of $Z$ are unchanged.

- $\quad$ upper right side: two sides views of the unexposed surface shape with a ratio $Z / X=Z / Y=4$.

- $\quad$ lower left side: top view of the unexposed surface temperature.

- $\quad$ lower right side: temperature and $Z$ value of the centre point of the unexposed surface respectively plotted in blue and red curves as a function of time. The vertical line indicates the time step of the $3 \mathrm{D}$ plots (the time value is also visible next to the stopwatch icon). 


\section{Metallic material}

The first preliminary tests were conducted on metallic test coupons. The materials and structures department (DMAS) of ONERA had extensively characterized the material properties and mechanical behaviour laws of the INCONEL 600. As a consequence, such test can easily be used for the validation of aero-thermo-mechanical numerical simulations involving flame/structure interactions on a nonreactive material. The test coupon is coated with a high emissivity black paint to perform quantitative and accurate IR thermography measurements. Moreover, the reflective coefficient of the raw material is too high to absorb the radiative contribution of the heat flux generated by the burner flame. Before being tested, the coated coupon then undergoes a heat treatment to avoid any off-gassing or detachment of the paint during the experiment.

The quality of the results from the image correlation was surprisingly good from the very first performed test despite the extreme thermal environment induced by the flame. The 3D reconstruction is applied successfully on the major part of the material surface. Few blank pixels are observed because a blanking threshold is activated if the DIC correlation is too low. This is particularly noticeable in the borders of the material as well as in some locations at the centre or at the corners. Adjusting level number of the pyramid has a significant effect on the correlation score as depicted in figure 5. The size of IW and the iteration number must be optimized throughout the experiment according to the image resolution and the texture quality from the light projection onto the material surface.

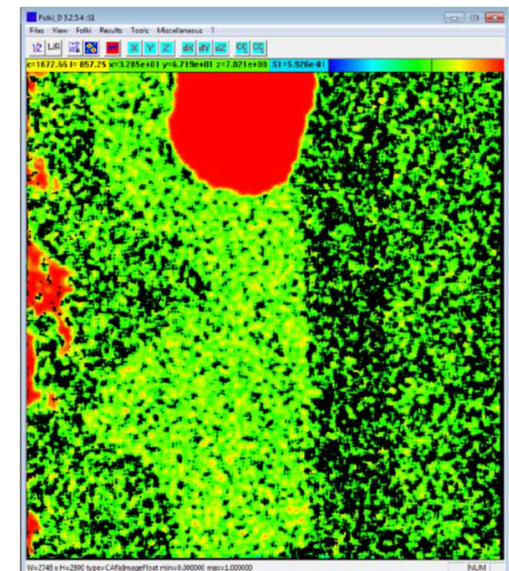

(a) Level $L=5$

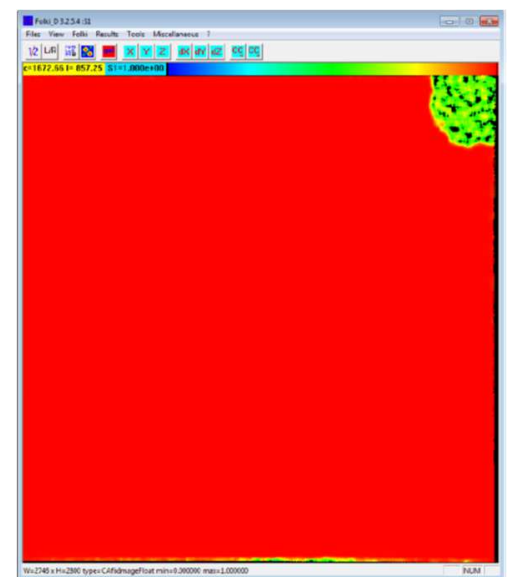

(b) Level $L=4$

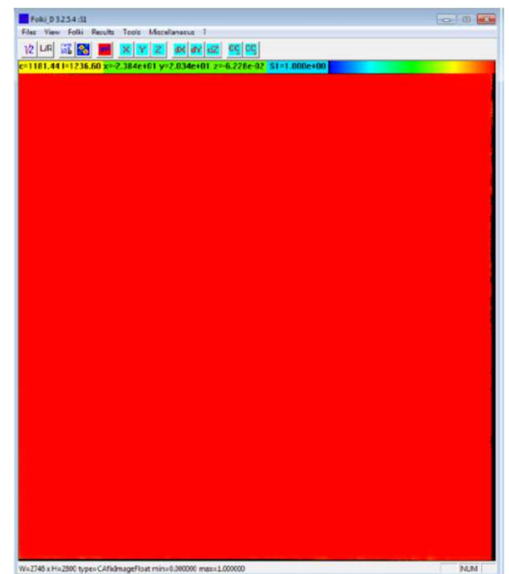

(c) Level $L=3$

Figure 5 - Effect of the level number $L$ of the pyramid on the correlation score

Results are presented in figure 6 . The test coupon is exposed to fire for $300 \mathrm{~s}$. The temperature at the centre of the unexposed surface increases up to $911 \mathrm{~K}$ before the burner is moved away. The maximum vertical displacement of the surface is reached at $150 \mathrm{~s}$ of the experiment with $Z=-16 \mathrm{~mm}$ from the initial position.

\section{Composite laminate}

Tests were then conducted on composite laminates with the same material used in the previous analyses performed with TGA and BLADE experiments ${ }^{1,2,3}$. The test coupon is uncoated to avoid any interference with the material natural reaction to fire. The emissivity and absorptivity of both surfaces are measured using a spectrophotometer as a function of the wavelength following the previously described procedure to accurately convert the luminance signal from the IR camera into temperature with the calibration law. The coupon has a rough surface and a smooth surface. The latter is exposed to fire and optical properties were characterised on both faces. The shape is initially flat. Results are presented in figure 7. 

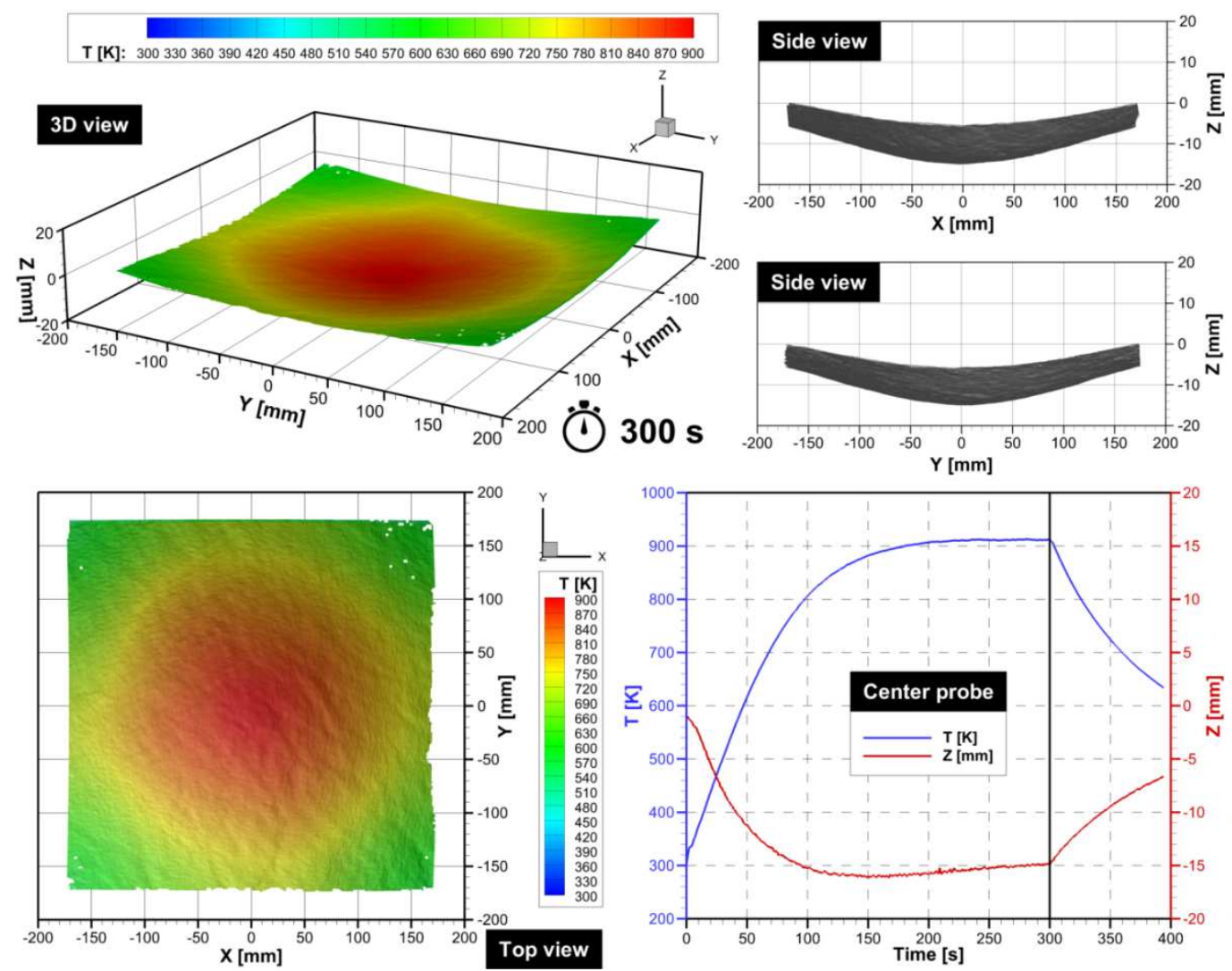

Figure 6-3D temperature reconstruction on the unexposed surface of an INCONEL 600 test coupon after $300 \mathrm{~s}$ of fire exposure

The test coupon is exposed to fire for $30 \mathrm{~s}$. The temperature at the centre of the unexposed surface increases up to $521 \mathrm{~K}$ at $t=20 \mathrm{~s}$ of fire exposure. Then measured temperature suddenly decays while the flame is still impacting onto the material exposed surface. The phenomenon is the thermal signature of the onset of delamination damage that induces a decrease of heat transfer in the through-thickness direction. The crack originates from the centre of the coupon unexposed surface and grows towards the borders. Concurrently, the crack thickness increases and significant swelling is observed in figure 7 at $t=30 \mathrm{~s}$. The centre point moved at $Z=+7.5 \mathrm{~mm}$ from the initial position. The onset of delamination also affects the symmetry of the stacking sequence of the laminate and the relaxation of internal stress induces a buckling effect as a consequence. It results in a dramatic flexural buckling at $t=37 \mathrm{~s}$ with $Z=+15.9 \mathrm{~mm}$ measured at the centre point $7 \mathrm{~s}$ after the end of the fire exposure. The unexposed surface, while highly delaminated, remains undecomposed at the end of the experiment. The thermal insulation induced by the crack layer provides protection for the backside of the material where the temperature stays below the pyrolysis onset temperature measured by TGA experiments ${ }^{3}$.

The 3D reconstruction is applied successfully on the major part of the material surface. However, more blank pixels are observed in this case because the rough surface distorts the speckle pattern projected by the LED and decreases the correlation score.

It is important to notice that the results presented in figures 6 and 7 were not adjusted, smoothed or filtered to eliminate any noise or any measurement bias. 

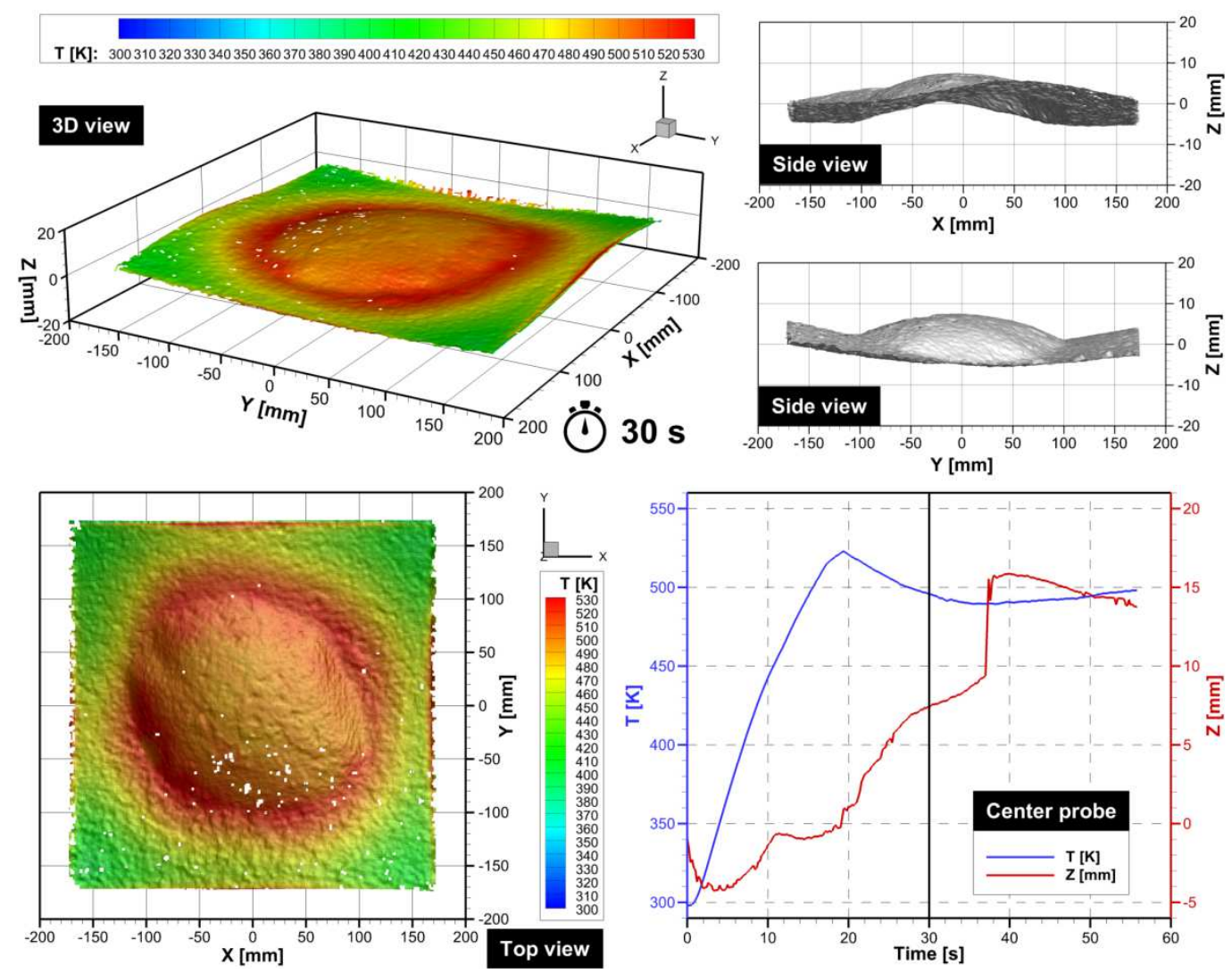

Figure 7 - 3D temperature reconstruction on the unexposed surface of a T700GC/M21-ISO8 test coupon after $30 \mathrm{~s}$ of fire exposure

\section{CONCLUSIONS}

Combining DIC and IR thermography measurements into a time-resolved 3D technique makes correlation between thermal response and mechanical behaviour very easy to investigate the materials reaction to fire. To remain non-intrusive, LED projection of the DIC pattern was preferred. A drawback is that neither the displacement nor the deformation can be assessed. Only the 3D shape can be measured because the illumination method is insensitive to in-plane displacement. The method was successfully applied for both metallic and composite materials exposed to fire despite the complex behaviour of composite laminates with the onset and growth of delamination damage and some off-gassing on the unexposed surface (figure 8(a)).

This study was an opportunity to bridge gaps between approach coming from different scientific communities by applying a methodology developed for fluid dynamics measurements and analysis tools to structure mechanics and fire sciences. Both optical set-up and FOLKI algorithm were inherited from fluid dynamics and heat transfer developments performed at ONERA with very good results and stateof-the-art quality. Using FOLKI algorithm instead of a commercial software gave the opportunity to implement temperature into the 3D shape reconstruction very easily. However, speckle projection studies are plethoric for profilometry and 3D reconstruction measurements ${ }^{12,13,14}$. FOLKI_D was derived from FOLKI_SPIV and was initially developed for measuring displacements and deformations on materials exposed to mechanical loading. The 3D shape reconstruction applied with a projected pattern is just a feature provided by FOLKI_D and used for this study. Therefore, other techniques exist ${ }^{12,13,14}$ and may provide results that are more accurate. Actually, if the magnitude of the measured displacement increases beyond the values measured in this study, FOLKI_D may have difficulty assessing the 3D shape. Current developments have thus separated the shape reconstruction from the displacement computation within the algorithm in order to be able to use other shape reconstruction methods if desired in future applications.

On-going improvements also consists in taking into account thermal effects affecting the DIC images 
at high temperature: thermal radiation of hot surfaces in the visible spectrum (figure 8(b)) and mirage effect induced by unsteady thermal plume developing above the material surface (granular or wavy surface effect particularly noticeable in results presented in figure 8). Extension of the method in the side exposed to fire is also considered using narrow band illumination and matched image filtering ${ }^{15}$.

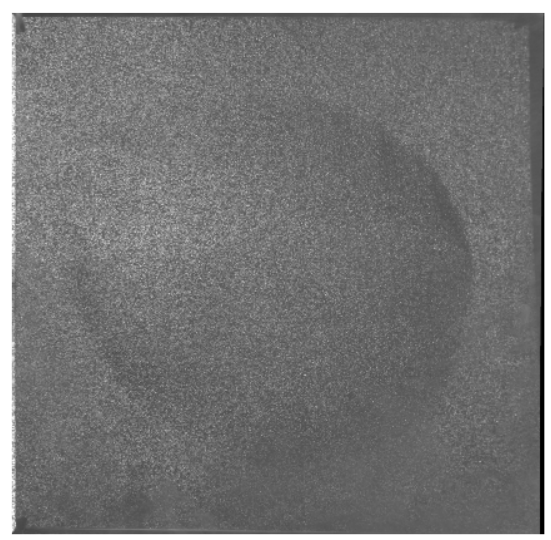

(a) Pyrolysis, delamination, off-gassing

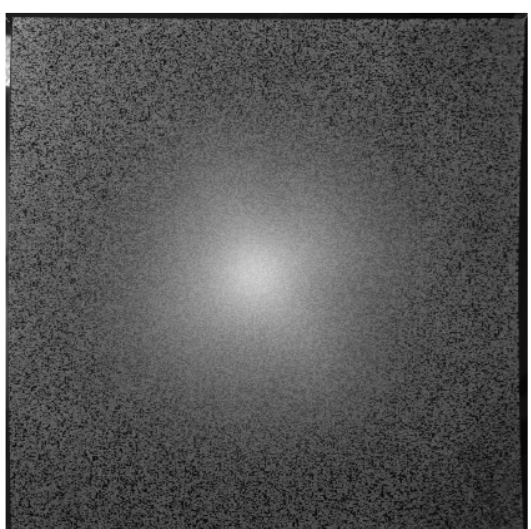

(b) Surface radiation, buoyancy

Figure 8 - Thermal effects on DIC

\section{Acknowledgments}

The FIRE facility has been developed within the frame of PhyFIRE project funded by the DGAC (Civil Aviation General Directorate) since 2016. Combining DIC and IR thermography measurements was done in ThermoSolEx project internally funded by ONERA. The test campaign on composite laminates was conducted in P7 project "Mitigating risk of fire, smoke and fumes" of Future Sky Safety European research programme (H2020 grant agreement No 640597).

\section{REFERENCES}

1. G. Leplat, V. Biasi, C. Huchette and P. Beauchêne. Extrapolation of thermochemical kinetics from conventional thermogravimetric analysis at very high heating rates for composites. In proceedings of FRPM17 - Fire Retardant Polymeric Materials, Manchester, UK, 2017.

2. G. Leplat, C. Huchette, A. Mavel and P. Nunez. Analysis of delamination onset and growth induced by laser decomposition within carbon/epoxy composite laminates. In proceedings of the $14^{\text {th }}$ International Conference on Fire Science \& Engineering INTERFLAM, London, UK, 2016.

3. G. Leplat, C. Huchette and V. Biasi. Thermal and Damage Analysis of Laser-Induced Decomposition within Carbon/Epoxy Composite Laminates. Journal of fire sciences, pp. 1-24, May, 2016. DOI: $10.1177 / 0734904116653617$.

4. J. Gales, L. Robertson and L. Bisby. Creep of prestressing steels in fire. Fire and Materials. 40:875-895, 2016.

5. N. Cholewa, P.T. Summers, S. Feih, A.P. Mouritz, B.Y. Lattimer, and S.W. Case. A technique for coupled thermomechanical response measurement using infrared thermography and digital image correlation (TDIC). Experimental Mechanics, 56(2):145-164, 2016.

6. J.L. Hodges, C.M. Rippe, S.W. Case, and B.Y. Lattimer. Thermographic digital image correlation (TDIC) measurements of mechanically-loaded structures with fire in the field of view. In proceedings of the $9^{\text {th }}$ International Conference on Structures in Fire, pages 811-818, 2016.

7. J.L. Hodges, C.M. Rippe, N. Cholewa, P.T Summers, Z. Mathys, A.P. Mouritz, S.W. Case and B.Y. Lattimer. Resolving obstructed views using multiple thermography digital image correlation systems. In proceedings of the $14^{\text {th }}$ International Fire Science \& Engineering Conference, pages 47-57, 2016.

8. B. Lucas and T. Kanade. An iterative image registration technique with an application to stereovision. In proceedings of $7^{\text {th }}$ International Joint Conference on Artificial Intelligence (IJCAI), pp. 674-679, 1981.

9. G. Le Besnerais and F. Champagnat. Dense optical flow estimation by iterative local window registration. in Proceedings of IEEE ICIP'05, vol. 1, pp. I-137-40, Genoa, Italy, Sept. 2005. 
10. F. Champagnat, A. Plyer, G. Le Besnerais, B. Leclaire, S. Davoust and Y. Le Sant. Fast and accurate PIV computation using highly parallel iterative correlation maximization. Experiments in fluids, vol. 50(4), p. 1169, 2011.

11. G. Le Besnerais, Y. Le Sant and D. Lévêque. Fast and dense 2D and 3D displacement field estimation by a highly parallel image correlation algorithm. Strain, vol. 52(4), pp. 286-306, 2016.

12. Pan, Bing, et al. Improved speckle projection profilometry for out-of-plane shape measurement. Applied optics. 47(29):5527-5533, 2008.

13. Wang, Zhaoyang, et al. Three-dimensional shape measurement with a fast and accurate approach. Applied optics. 48(6):1052-1061, 2009.

14. Zhu, Feipeng, et al. Accurate 3D measurement system and calibration for speckle method. Optics and Lasers in Engineering. 48(11):1132-1139, 2010.

15. C. M. Smith and M. S. Hoehler. Imaging Through Fire Using Narrow-Spectrum Illumination. Fire Technology, vol. 54, pp. 1705-1723, 2018. 